\title{
Interoception drives increased rational decision-making in meditators playing the ultimatum game
}

\author{
Ulrich Kirk', Jonathan Downar ${ }^{2,3}$ and P. Read Montague ${ }^{1,4 *}$ \\ 1 Human Neuroimaging Laboratory, Virginia Tech Carilion Research Institute, Virginia Tech, Roanoke, VA, USA \\ 2 Neuropsychiatry Clinic, Toronto Western Hospital, Toronto, ON, Canada \\ 3 Centre for Addition and Mental Health, University of Toronto, Toronto, ON, Canada \\ ${ }^{4}$ Department of Physics, Virginia Tech, Blacksburg, VA, USA
}

\section{Edited by:}

Julia Trommershaeuser, New York

University, USA

\section{Reviewed by:}

Kerstin Preuschoff, University of Zurich, Switzerland

Jill X. O'Reilly, University of Oxford, UK Daniel Campbell-Meiklejohn, Aarhus

University, Denmark

\section{${ }^{*}$ Correspondence:}

P. Read Montague, Human

Neuroimaging Laboratory, Virginia Tech Carilion Research Institute, 2 Riverside

Circle, Roanoke, VA 24016, USA.

e-mail: read@vt.edu
Human decision-making is often conceptualized as a competition between cognitive and emotional processes in the brain. Deviations from rational processes are believed to derive from inclusion of emotional factors in decision-making. Here, we investigate whether experienced Buddhist meditators are better equipped to regulate emotional processes compared with controls during economic decision-making in the Ultimatum Game. We show that meditators accept unfair offers on more than half of the trials, whereas controls only accept unfair offers on onequarter of the trials. By applying $\mathrm{fMRI}$ we show that controls recruit the anterior insula during unfair offers. Such responses are powerful predictors of rejecting offers in social interaction. By contrast, meditators display attenuated activity in high-level emotional representations of the anterior insula and increased activity in the low-level interoceptive representations of the posterior insula. In addition we show that a subset of control participants who play rationally (i.e., accepts $>85 \%$ unfair offers) recruits the dorsolateral prefrontal cortex presumably reflecting increased cognitive demands, whereas rational meditators by contrast display elevated activity in the somatosensory cortex and posterior superior temporal cortex. In summary, when assessing unfairness in the Ultimatum Game, meditators activate a different network of brain areas compared with controls enabling them to uncouple negative emotional reactions from their behavior. These findings highlight the clinically and socially important possibility that sustained training in mindfulness meditation may impact distinct domains of human decision-making.

Keywords: decision-making, fMRI, mindfulness, posterior insula, anterior insula, social fairness, DLPFC, striatum

\section{INTRODUCTION}

In rational accounts of human behavior, if a person is offered the choice of gaining a reward versus gaining nothing, they should always choose the reward. While this is typically true in a nonsocial context, this account often breaks down during social interactions. In the classic example of the Ultimatum Game, a "proposer" offers to split a sum of money with a "responder" in a two-person exchange. If the responder rejects the offer, both players get nothing - hence, according to rational choice theory, responders should accept all non-zero offers. In reality, players are rarely so magnanimous. Responders typically reject offers in which the proposer's share exceeds $80 \%$ of the total, preferring to gain nothing rather than accept an inferior share of the winnings (Guth et al., 1982; Bolton and Zwick, 1995). This sensitivity to fairness may be a uniquely human trait (Fehr and Fischbacher, 2003). For example, chimpanzees play the Ultimatum Game according to the dictates of rational choice theory, and are content with all non-zero offers irrespective of fairness (Jensen et al., 2007).

So why do human beings turn a perfectly good reward into a disappointment when others are getting more? One proposal is that the superficially "irrational" rejection of inferior shares is a costly but effective means of enforcing social norms (Boyd et al., 2003). Ultimately, the costs of giving up inferior shares are presumably outweighed by the long-term benefits of establishing a cooperative social environment (Fehr and Gachter, 2002; de Quervain et al., 2004). Although this may be so, the strategy of measuring one's own gains against others also has a downside. In wealthy societies, a comfortable income can become unsatisfactory if compared to an extravagant one: the millionaire envies the billionaire, and the billionaire envies still richer billionaires. This arms race of reward can in itself lead to inequity and social unrest, as in the rapid escalation of corporate executive compensation in recent years, which led to a backlash of widespread public indignation (Ariely, 2008).

The human tendency to see rewards in socially relative terms may therefore be a mixed blessing. If so, the question arises as to whether some people are capable of ignoring social considerations and assessing a reward based on its intrinsic qualities alone. The willingness to sacrifice money to punish an unfair proposer is associated with affective responses, as measured by skin conductance responses (van't Wout et al., 2006). These affective responses have specific neural correlates, identified in neuroimaging studies of social exchange. Unfair offers elicit greater activation in the anterior insula (Rilling et al., 2002; Sanfey et al., 2003; King-Casas et al., 2008), an area linked to interoceptive function (Craig, 2002, 2009; Critchley et al., 2004), and in particular to the emotion of disgust (Calder et al., 2001). Anterior insula activation scales inversely with offer size, and predict whether an unfair offer will 
be rejected (Sanfey et al., 2003). These results suggest that rejection of unfair offers in the Ultimatum Game is driven by negative emotional reactions.

If some individuals had expertise in uncoupling such emotional reactions from their actual behavior, they might be better equipped to assess a reward on its own merits. Such individuals could potentially be found among experienced practitioners of mindfulness meditation. Mindfulness meditation has its roots in a 2500-year-old Buddhist tradition, and has grown increasingly popular in Western societies over the last few decades. A central technique in mindfulness is to attend to the events in the present moment, on purpose, in a spirit of observation rather than judgment.

Meditation has been conceptualized as complex emotional regulatory training techniques developed for cultivation of well-being and emotional balance (Lutz et al., 2008a). Previous research on emotion regulation show that humans are able to exert a degree of control by reducing or enhancing emotional reactions elicited by various categories of stimuli and events in the world (Jackson et al., 2000; Ochsner et al., 2002; Ochsner and Gross, 2005; Eippert et al., 2007). Based on these previous studies we hypothesized that successful regulation of negative emotional reactions would lead to increased acceptance rates of unfair offers in the Ultimatum Game. In line with this hypothesis, we expected that meditators would exhibit greater acceptance rates for highly asymmetric offers.

Recent neuroimaging studies suggest some convergence between the neural correlates of social exchange and mindfulness meditation. Voxel-based morphometry studies demonstrate higher gray matter volumes in the anterior insula of meditators versus agematched controls, suggesting long-term effects of meditation (Lazar et al., 2005; Holzel et al., 2008; Luders et al., 2009). The mid and posterior aspects of the insular cortex are activated during meditative states (Farb et al., 2007; Lutz et al., 2008b, 2009). In addition, even a short course of mindfulness training is effective in decoupling the activity of the insula from the activity of other regions involved in social decision-making, such as the medial prefrontal cortex (Farb et al., 2007; Tang et al., 2009). Taken together, these findings suggest that experienced mindfulness meditators could be capable of regulating emotional and social considerations in their evaluation of rewards in the Ultimatum Game.

Specifically, we hypothesized that the key anatomical locus for such regulation would be attenuation of the anterior insula responses toward unfair offers in meditators compared with controls. Furthermore, we expected that meditators would attend to internal bodily states in order to successfully regulate negative emotional reactions to unfair monetary offers. In accordance with previous studies we expected that elevated interoceptive awareness would involve brain regions such as the posterior parts of the insula and somatosensory cortices (Farb et al., 2007; Lutz et al., 2008b, 2009; Tang et al., 2009). Conversely, we expected that non-meditator controls might rely more on medial cortical areas linked to prospection and theory of mind, in line with previous studies (Rilling et al., 2002; Sanfey et al., 2003).

To test these hypotheses, we recruited a group of experienced mindfulness meditators and a control group of non-meditators. We enrolled subjects in the study to play the role of responders in an anonymous version of the Ultimatum Game (Figure 1), while undergoing fMRI. We report both the behavioral and the neuroimaging findings resulting from the experiment.

\section{MATERIALS AND METHODS SUBJECTS}

Sixty-six subjects participated in the study. Subjects were recruited in two groups. One group $(n=40)$ consisted of controls ( 21 females $/ 19$ males). The second group $(n=26)$ consisted of expert meditators (10 females/16 males). Practitioners had various degrees of regular experience ranging from 6 month to 24 years. Common to all practitioners were that they practiced Buddhist meditation while maintaining a secular life incorporating a career, family, and friends. See Table 5 for comparison of demographic variables. It should be emphasized that having more control subjects $(n=40)$ than meditators $(n=26)$ does not compromise the validity of the behavioral or neural tests, on the contrary, this improves our power for detecting any between group differences by decreasing the standard error on the characterization of the response profile for controls. All subjects had normal or corrected-to-normal vision, and none had a history of neurological or psychiatric disorders, and no current use of psychoactive medications. All procedures were conducted in accordance with the Institutional Review Board at Baylor College of Medicine. Both groups completed pre-scan questionnaires to assess potential differences between groups. There were no significant differences between groups on Beck Anxiety Inventory (two sample $t=-1.63 ; \mathrm{df}=64 ; p<0.1$ ), and Beck Depression Inventory (two sample $t=0.67 ; \mathrm{df}=64 ; p<0.5$ ). As expected significant differences emerged between groups as assessed by two mindfulness questionnaires: Mindfulness Attention Awareness Scale (MAAS; Brown and Ryan, 2003; two sample $t=2.70 ; \mathrm{df}=64 ; p<0.008)$, and Kentucky Inventory of Mindfulness Skills (KIMS; Baer et al., 2004; two sample $t=2.47$; $\mathrm{df}=64 ; p<0.01)$.

\section{EXPERIMENTAL PROCEDURES}

Participants played responders during 45 rounds of an anonymous version of the Ultimatum Game. Prior to scanning, participants were instructed in the task and were subsequently given a test to ensure that the nature and rules of the game were comprehensible to all participants. The offers were splits of $\$ 20$. On each round, the participants saw a bar graph with an offer (e.g., "Tom proposes: \$9 you \$11 Tom"; Figure 1B). The offer screen had a duration of $6 \mathrm{~s}$. Next the participants were presented with the choice: "Accept (\$9) Reject (\$0)," which was presented for $3 \mathrm{~s}$ in which subjects made a response using a button-box. A red box placed around one of the choices indicated that a decision was made. Finally, a jittered inter-trial interval was presented (2-4 s). Participants had a button-box in each hand and were instructed to press with either left or right hand corresponding to the preferred choice, which was presented on left and right side of the screen. Position of the "accept" and "reject" choices on either left or right side was held constant within subjects, and counterbalanced across subjects. Thirty rounds were played with human partners and 15 with computer partners. Participants received offers in a predetermined fashion: $6 \times \$ 19: 1,6 \times \$ 18: 2,6 \times \$ 17: 3,6 \times \$ 16: 4$, $6 \times \$ 15: 5,3 \times \$ 14: 6,3 \times \$ 13: 7,3 \times \$ 12: 8,3 \times \$ 11: 9,3 \times \$ 10: 10$. The sequence of offer presentations was randomized across participants. Participants were presented with an identical range of offers in human and computer rounds. 


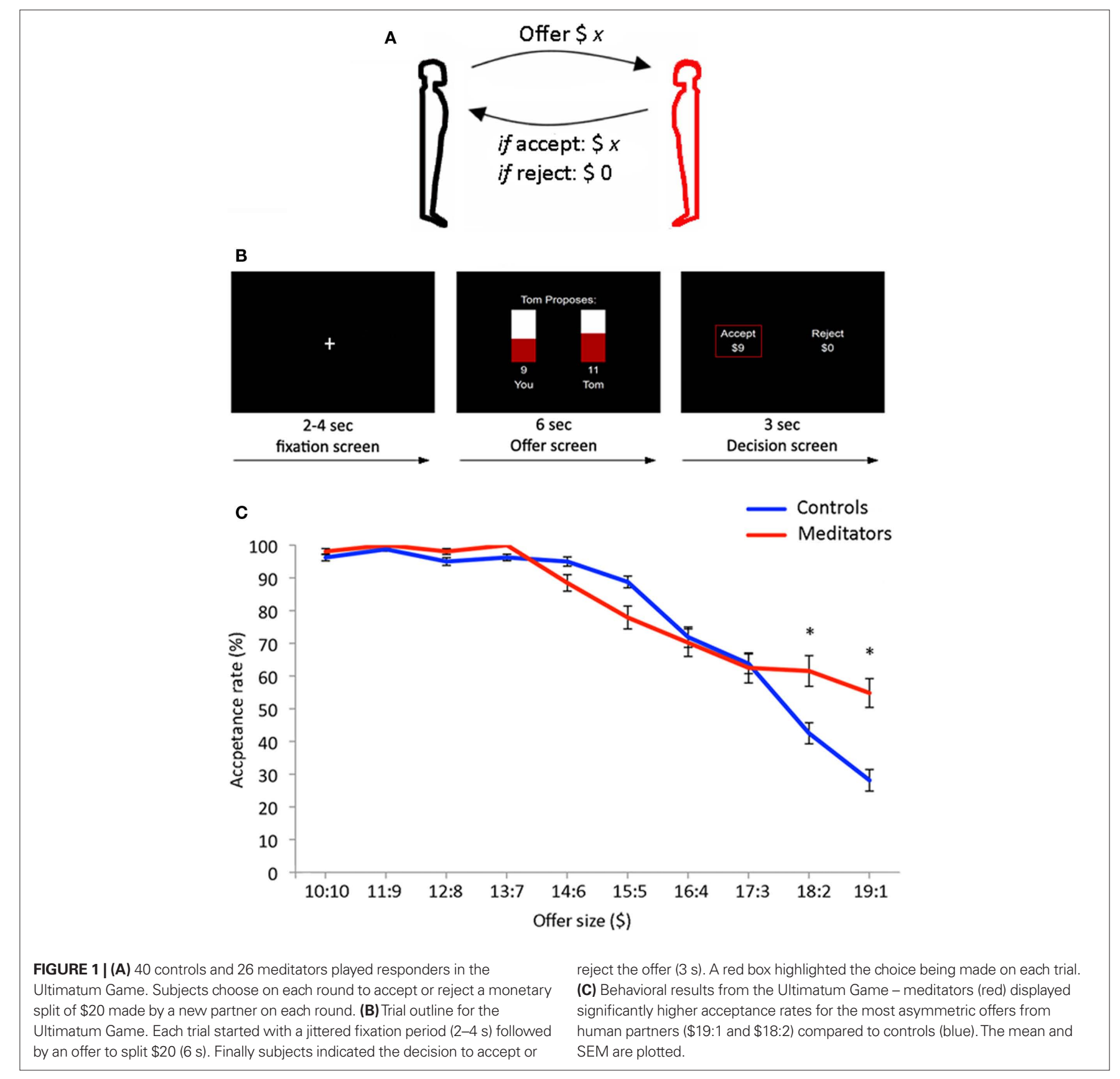

The stimuli were presented at a screen resolution of $1024 \times 768$ pixels. Stimuli were presented and responses collected using NEMO (Human Neuroimaging Lab, Baylor College of Medicine). The stimuli were back-projected via an LCD projector onto a transparent screen positioned over the subjects' head and viewed through a tilted mirror fixed to the head coil.

Prior to the experiment participants were told that the offers presented in the human rounds had been made by proposers in a previous experiment, i.e., that the offers were real, and that proposers would be paid according to the decision made by the participants. It was made explicit to participants that they would play a different person on each round. This served as a cover story to enhance the ecology of the game, i.e., that the choices made by participants indeed had consequences for partners on each round of the game. In reality, all the proposals were predetermined similar to other neuroimaging studies using the Ultimatum Game (Sanfey et al., 2003; Knoch et al., 2006; Koenigs and Tranel, 2007; Crockett et al., 2008).

A categorization of fair and unfair offers was made based on empirical data from previous studies of Ultimatum Game responses showing that offer sizes above 30\% of the total sum are typically accepted (Guth et al., 1982; Thaler, 1988; Novak et al., 2000). The data in the present study displayed similar acceptance ratios. Proposals of $30 \%$ of the total sum (corresponding to the $\$ 14: 6$ offer sizes in the present study) displayed acceptance rates of $88 \%$ ( std $=5.04$ ) for meditators, and $95 \%$ 
( std $=2.99)$ for controls for human conditions. These two lines of evidence support a categorization of fair (\$10:10-\$14:6) and unfair (\$19:1-\$15:5) offers.

At the end of the experiment, participants were paid according to the decisions they made on one randomly selected round. In addition participants were paid a flat fee of $\$ 20$ (to cover parking expenses and compensation for completing paperwork). Participants were informed about this payment method prior to the experiment.

\section{fMRI DATA ACQUISITION}

The anatomical and functional imaging was performed using 3 Tesla Siemens Trio scanners. High-resolution T1 weighted scans were acquired using an MPRAGE sequence (Siemens). Functional imaging used an EPI sequence with a repetition time (TR) of $2000 \mathrm{~ms}$, echo time $(\mathrm{TE})=25 \mathrm{~ms}$, flip angle $=90^{\circ}, 220 \mathrm{~mm}$ field of view (FOV), $64 \times 64$ matrix. Functional slices were oriented $30^{\circ}$ superior-caudal to the plane through the anterior and posterior commissures in order to reduce signal drop-out due to magnetic field in-homogeneities (Deichmann et al., 2003). Each functional image was acquired in an interleaved way, comprising thirtyseven $4 \mathrm{~mm}$ axial slices for measurement of the blood oxygenation level-dependent (BOLD) effect (Ogawa et al., 1990), yielding $3.4 \mathrm{~mm} \times 3.4 \mathrm{~mm} \times 4.0 \mathrm{~mm}$ voxels.

\section{fMRI DATA ANALYSIS}

Image pre-processing and data analysis was performed using SPM2 (Wellcome Department of Imaging Neuroscience, London, UK). Motion correction to the first functional scan was performed using a six parameter rigid-body transformation (Friston et al., 1996). The average of the motion-corrected images was co-registered to each individuals structural MRI using a 12 parameter affine transformation. Slice timing artifact was corrected, after which images were spatially normalized to the Montreal Neurological Institute (MNI) template provided in SPM2. Images were then spatially filtered with an 8-mm isotropic Gaussian kernel and for the analysis a high pass filter with a cut-off frequency at $1 / 128 \mathrm{~Hz}$ was applied.

Following pre-processing a general linear model (GLM) was applied to the fMRI time-series that time-locked single impulse response functions at offer onset including the 6-s epoch following the offer. The model included 20 regressors, which modeled the 10 human offer conditions and the 10 computer offer conditions. Results from the computer conditions will be summarized in a separate paper. Residual effects of head motion were corrected for by including the six estimated motion parameters for each subject as regressors of no interest. The model was convolved with the canonical hemodynamic response function (HRF; Friston et al., 1998). The mean images from the first-level analysis were entered into a second-level, random effects (RFX) analysis accounting for the between subject variance. An ANOVA model using the beta estimates of the regressors of interest was used. Equal variance was not assumed, thus SPM2's options for non-sphericity correction was applied (Glaser and Friston, 2004). Using $t$-contrasts allowed us to test for correlations of the fMRI BOLD signal and the parameters of interest. The resulting $t$ maps were subsequently transformed to the unit normal $z$-distribution to create a statistical parametric map for each contrast. The statistical results given were based on a single-voxel $t$-statistic or cluster-level corrected corresponding to $p<0.05$ corrected for multiple comparisons with an extent threshold of $>10$ voxels (unless otherwise stated). Where cognitive conjunctions were used a threshold of $p<0.001$, uncorrected was applied. The coordinates of all activations are reported in MNI space.

A ROI analysis in bilateral anterior insula was identified using the coordinates provided by a previous study (Sanfey et al., 2003). Coordinates from this study were transformed from Talairach space to MNI space (www.mrc-cbu.cam.ac.uk). A spherical mask with a 5-mm radius centered at [35 154 ) and [ $\left.\begin{array}{llll}-33 & 14 & 0\end{array}\right]$ was used to extract the time-series from bilateral anterior insula for human trials. A correlation analysis was computed to explore if the anterior insula co-varied with individual differences in terms of the percent acceptance rates for the most unfair offer conditions (\$19:1 and $\$ 18: 2$ ) for each group separately. The first-level beta values using the regressors of interest (\$19:1 and \$18:2) were averaged and entered into a regression analysis at the second level.

A second ROI analysis was performed for bilateral anterior insula using identical parameters as in the previous ROI except that both fair (i.e., \$10:10-\$14:6) and unfair offer sizes (i.e., \$19:1-\$15:5) were included in the analysis. Consistent with previous models the offer onset was modeled as single impulse response functions including the 6-s epoch following the offer. Beta values within this ROI were extracted and mean \pm SE were computed for each group and plotted in fair and unfair bins.

In order to explore if unfair human offers differed according to the decision to accept or reject an unfair offer, we separated the data into subsequent decision to accept or reject unfair human offers. Specifically, using the coordinates from the interaction analysis for human trials [Meditators Unfair > Meditators Fair] $>$ [Controls Unfair $>$ Controls Fair] centered in the right posterior insula [48 -28 20] constituting 29 voxels, we extracted the mean \pm SE beta values within this region according to subsequent decision.

We performed a linear regression (separately for the two groups) to explore individual differences in trait mindfulness levels as measured by the MAAS and the KIMS. We regressed a behavioral measure of the size of the MAAS and KIMS for each individual on a neural measure of the impact on posterior insula activity. Specifically, the neural measure was given by the estimated beta value at peak voxels from the right posterior insula derived from the contrast [Meditators Unfair > Meditators Fair] $>$ [Controls Unfair > Controls Fair]. Each individual was an observation.

\section{BEHAVIORAL RESULTS}

We first asked whether meditators were less likely than controls to reject opportunities for monetary gain during asymmetric offers in the Ultimatum Game when playing with human partners. In accordance with previous findings (Guth et al., 1982; Bolton and Zwick, 1995; Sanfey et al., 2003), both meditators and controls tended to accept offers that were relatively symmetrical ( $\$ 10: 10$ $\$ 14$ :6; Figure 1C). Likewise, both groups showed an increasing tendency to reject monetary rewards as the offers became more asymmetric, overall (\$15:5-\$19:1). However, the two groups showed a bifurcation of acceptance rates for the most asymmetric offers (\$18:2 and \$19:1). Compared to controls, meditators were significantly less likely to reject opportunities for monetary gain 
for the two most asymmetric offer conditions ( $\$ 19: 1$; two sample $t=2.44$, df $=64, p<0.01$. \$18:2; two sample $t=2.39$, df $=64$, $p<0.01)$. In absolute terms, meditators were willing to accept a $\$ 19: 1$ split on $54 \%$ of trials, while controls were willing to accept this same division on only $28 \%$ of trials. Similarly, meditators were willing to accept an $\$ 18: 2$ split on $61 \%$ of trials, while controls were willing to accept this split on only $42 \%$ of trials. Hence, for the most asymmetric offers, meditators were more likely than controls to accept an opportunity for personal monetary gain, notwithstanding the larger gains accruing to their partners on these trials.

\section{NEUROIMAGING RESULTS}

Our behavioral findings suggested a difference between meditators and controls in the response to asymmetric (unfair) offers. For each group, we therefore began by identifying the brain areas that showed a difference in activity during the offer period for unfair (\$15:5-\$19:1) versus fair (\$10:10-\$14:6) offers. In meditators, greater activation for unfair offers appeared in the bilateral posterior insula, right postcentral gyrus, ACC (BA 32), left mid- anterior insula, thalamus, and cerebellum (Table 2). By contrast, controls displayed greater activity in bilateral anterior insula/inferior frontal gyrus (IFG), bilateral medial frontal gyrus (BA 9/10), bilateral ACC (BA 24/32), bilateral middle temporal gyrus, right supramarginal gyrus, and cerebellum (Table 3 ). To test for common areas activated by unfairness in both meditators and controls, we performed a conjunction analysis. This analysis revealed a common response to unfairness in the left ACC (BA 24/32).

Having established the neural correlates of unfairness in each group, we performed a direct contrast of the neural responses to unfair offers between the two groups. In this contrast, controls showed significantly greater activity than meditators in the left dorsal striatum, left lingual gyrus, bilateral precuneus, and middle occipital gyrus (Figure 2; Table 1). Conversely, meditators showed significantly greater activity than controls in bilateral mid- and posterior insula and bilateral posterior parietal cortex (PPC; Figure 2; Table 1).

We next performed a contrast to identify brain regions showing a significant interaction between group (meditator versus control) and offer type (unfair versus fair). In this contrast, meditators displayed a different response profile from controls in the right postcentral gyrus and right posterior insula (Figure 3A; Table 4). Mean beta values for the activation in the right posterior insula scale linearly with unfairness in meditators (Figure 3C), but not in controls (Figure 3D). Furthermore, in meditators only, right posterior insula activity was predictive of the subsequent decision to accept or reject an unfair offer (Figure 3B). Meditators showed significantly greater activity in this region when unfair offers were later rejected (paired $\left.t=4.8 ; p<10^{-6}\right)$. In controls, no such distinction was apparent (paired $t=0.3 ; p<0.7$ ). The converse interaction displayed activity in the right supramarginal gyrus (Table 4). We estimated a linear regression of the impact of right posterior insula against a behavioral measure of each individual's score on two mindfulness scales. We employed the MAAS and KIMS as both of these scales has in previous studies been applied as a measure of trait levels of mindfulness (Brown and Ryan, 2003; Baer et al., 2004; Creswell et al., 2007). We performed the correlation separately for meditators and controls. The analysis showed that right posterior insula correlated with individual differences in trait mindfulness levels in the meditator group (MAAS; $R=0.55, p=0.001$. KIMS; $R=0.6, p=0.001$; Figures 3E,F). This correlation was absent in controls (MAAS; $R=0.18, p=0.1$. KIMS; $R=0.21, p=0.1$; Figure not shown).

Previous studies have associated activity in the anterior insula with responders' negative emotional response to an unfair offer (Sanfey et al., 2003). We therefore performed an ROI analysis in

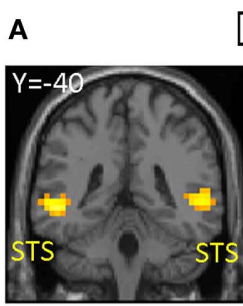

\section{[Ctr Unfair > Ctr Fair]}
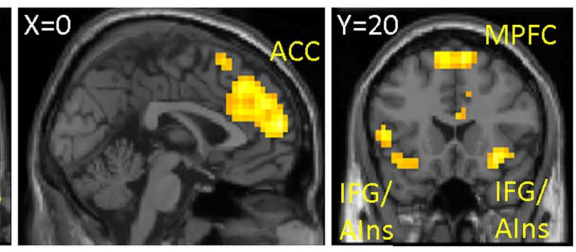

c

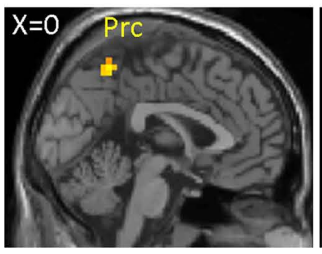

[Ctr Unfair > Medi Unfair]

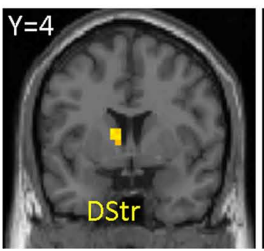

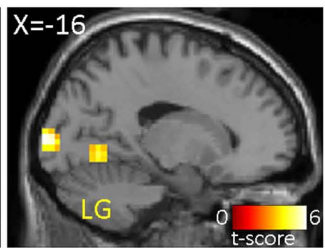

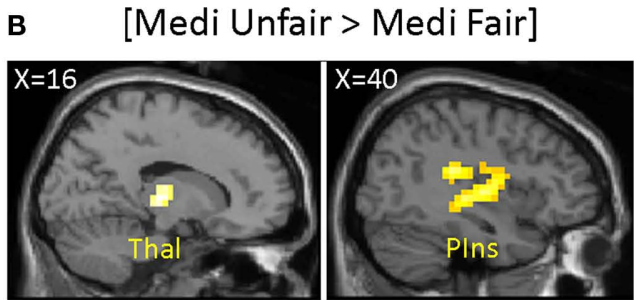

D [Medi Unfair > Ctr Unfair]
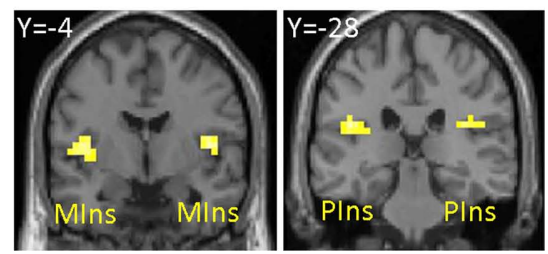

FIGURE 2 | Within group (A,B) and across group (C,D) main effects of unfair offers. (A) Controls (Ctr) displayed more activity in bilateral superior temporal sulcus (STS), anterior cingulate cortex (ACC), bilateral inferior frontal gyrus (IFG)/ anterior insula (Alns), and superior frontal gyrus (MPFC) for unfair offers relative to fair offers by human partners. (B) Significant activity to unfair versus fair offers was found in meditators (Medi) in the thalamus (Thal), bilateral posterior insula
(PIns), left mid-anterior insula, and ACC. Direct comparison between unfair offers across groups: (C) Left precuneus (Prc), left dorsal striatum (DStr), and lingual-occipital gyri (LG) are shown overlaid on coronal and sagittal sections. (D) Bilateral mid insula (MIns) and bilateral posterior insula (PIns) are shown on separate coronal sections. The activations are FDR-corrected. The ACC is displayed at $p<0.001$, uncorrected to illustrate the extent of the activation. 
Table 1 | Between-group main effects of unfair offers for human conditions.

\begin{tabular}{lcrrrrr}
\hline Region & Voxels & $\boldsymbol{t}$-Value & \multicolumn{3}{c}{ MNI } \\
\cline { 5 - 7 } & & & $\boldsymbol{x}$ & $\boldsymbol{y}$ & $\boldsymbol{z}$ \\
\hline [MEDITATORS UNFAIR > CONTROLS UNFAIR] & & & \\
Right mid/posterior insula & 14 & 4.20 & 44 & -4 & 8 \\
Left mid/posterior insula & 40 & 4.15 & -44 & -4 & 8 \\
Right posterior insula & 16 & 3.97 & 36 & -28 & 20 \\
Left posterior insula & 62 & 4.51 & -44 & -24 & 20 \\
Right posterior parietal cortex (PPC) & 23 & 4.05 & 20 & -64 & 40 \\
Left posterior parietal cortex (PPC) & 33 & 4.52 & -32 & -69 & 32 \\
[CONTROLS UNFAIR > MEDITATORS UNFAIR] & & & \\
Right middle occipital gyrus & 46 & 5.86 & 24 & -96 & 4 \\
Left middle occipital gyrus & 63 & 6.30 & -28 & -92 & 0 \\
Left lingual gyrus & 21 & 4.94 & -16 & -64 & -4 \\
Right precuneus & 11 & 4.38 & 16 & -76 & 28 \\
Left precuneus & 13 & 4.60 & -4 & -52 & 56 \\
Left dorsal striatum & 11 & 4.11 & -12 & 4 & 12
\end{tabular}

Activations are displayed at $p<0.05$, FDR-corrected. Extent threshold $>10$ voxels.

this region to assess its response to unfair offers in meditators and controls. Using the coordinates of the study cited above, we placed a 5-mm spherical ROI in the bilateral anterior insula and extracted beta estimates for fair and unfair offers in each group. In controls, the right and left anterior insula showed significantly higher activity for unfair versus fair offers (Left: paired $t=3.4, p<10^{-4}$. Right: paired $t=2.6, p<0.008)$. However, no such differences were seen in meditators (Left: paired $t=1.3, p<0.2$. Right: paired $t=0.7$, $p<0.4$; Figures $4 \mathrm{D}, \mathrm{E})$. Furthermore, left anterior insula displayed a significant difference between unfair offers in controls compared to meditators (two sample $t=1.9, p<0.05$ ). There were, however no such difference between unfair offers in controls compared to meditators in the right anterior insula (two sample $t=0.8, p<0.4$ ). Importantly, there was no difference in bilateral anterior insula between fair offers in the two groups.

We also sought to determine whether neural activity in the anterior insula ROIs could predict individual subjects' acceptance rates for the most unfair offers ( $\$ 19: 1$ and $\$ 18: 2$ ). Control subjects with stronger anterior insula activation for unfair offers showed lower acceptance rates for these offers (Left: $R=-0.41, p=0.004$, one-tailed. Right: $R=-0.45, p=0.002$, one-tailed; Figure 4B). However, in meditators, this was not the case. Neural activity in the anterior insula did not predict acceptance rates for unfair offers in the meditator group (Left: $R=-0.23, p=0.12$, one-tailed. Right: $\mathrm{R}=-0.31, p=0.06$, one-tailed; Figure $4 \mathrm{C}$ ).

We finally made a comparison between the participants who on average accepted $>85 \%$ of the most unfair offers ( $\$ 19: 1$ and $\$ 18: 2$ ). This process yielded a subdivision of the most rational participants from the control group $(n=9)$ and meditator group $(n=14)$. We expected that rational controls would recruit the DLPFC as previous studies have demonstrated that the DLPFC reduces subjects' willingness to reject unfair offers in the Ultimatum Game (Sanfey et al., 2003; Knoch et al., 2006). In a contrast between rational controls versus rational meditators we found elevated activity in bilateral DLPFC (Left: $z=3.15 ;-324828$; $p<0.001$, uncorrected. Right: $z=3.09$; 3648 20; $p<0.001$, uncorrected; Figure 5, left panel). In the opposite contrast [meditators $>$ controls] we did not observe activity in the DLPFC, but found activity in the left postcentral gyrus extending into the posterior insula $(z=4.51 ;-52$ -2040 ; $p<0.001$, uncorrected), the left posterior superior temporal cortex (pSTC; $z=3.65 ;-48-5612 ; p<0.001$, uncorrected), and bilateral parahippocampal gyrus (Left: $z=3.91 ; 32-40-16$. Right: $z=3.73 ;-28-40-16, p<0.001$, uncorrected; Figure 5, right panel).

\section{DISCUSSION}

A rational economic agent, homo economicus, should in theory accept all non-zero offers in the Ultimatum Game, since any amount of reward is better than nothing. However, in reality, human beings have a strong tendency to measure their rewards against the rewards of their peers. This tendency is often described as a characteristically human form of irrational behavior (Fehr and Fischbacher, 2003). Yet in this study, we identified a population of human beings who play the Ultimatum Game more like homo economicus. Experienced meditators were willing to accept even the most asymmetrical offers on more than half of the trials, whereas control members of homo sapiens did so just over one-quarter of the trials.

In dual-process accounts of human decision-making, separate "rational," and "emotional" systems compete to control the outcome of human decisions (Tversky and Kahneman, 1981; St. Evans, 2008). Neuroimaging studies have suggested that these two systems may have distinct neural correlates. For example, intuitive or emotion-driven decisions tend to be associated with greater activity in medial prefrontal and medial orbitofrontal cortical areas and their striatal counterparts, while more rational or deliberative decisions tend to be associated with a shift in activity toward more lateral prefrontal and parietal areas and their striatal counterparts (Goel and Dolan, 2003; Greene et al., 2004; McClure et al., 2004). Hence, when comparing meditators to control subjects, we might have expected to see a shift in activity from medial to lateral prefrontal cortical areas in meditators playing the Ultimatum Game. In reality, the neuroimaging results showed quite a different pattern. During decisionmaking, control subjects activated a network of areas including medial prefrontal cortex, anterior cingulate cortex, and superior temporal sulcus (Figure 2A; Table 3). This network was largely consistent with that seen in previous neuroimaging studies of the Ultimatum Game, and other tasks involving social cognition and theory of mind (Sanfey et al., 2003; Amodio and Frith, 2006; Adolphs, 2009). In sharp contrast, meditators showed activity in an entirely separate network, which comprised primarily the mid- and posterior insula and ventral posterior thalamus (Figure 2B; Table 2). Rather than social cognition and theory of mind, these areas are more typically associated with interoception: the representation of the body's internal state (Craig, 2002, 2009). Specifically, a representation of the body's internal state is mapped by afferents through the ventromedial thalamic nucleus to the sensorimotor cortex and the mid/posterior insula (Craig, 2002).

Strikingly, there was very little overlap in activity between meditators and controls. The left ACC (BA 24/32) was the only region activated in a conjunction analysis between controls and medi- 
A

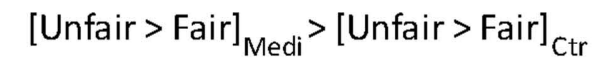

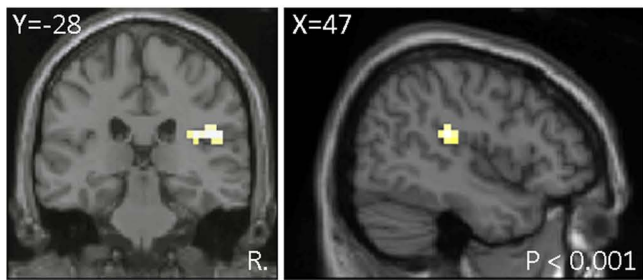

Meditators Controls

C

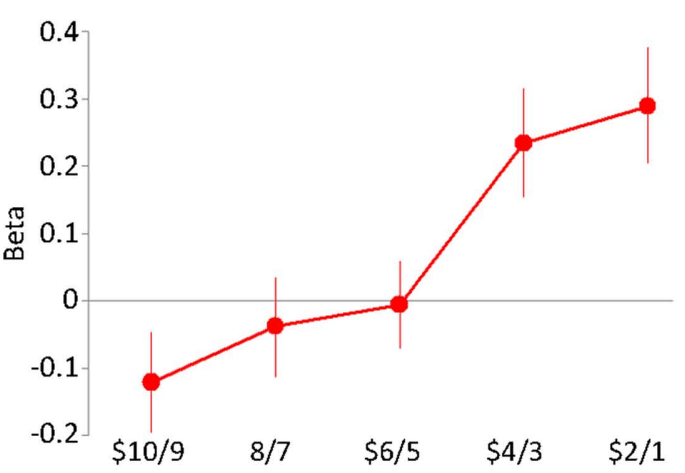

E

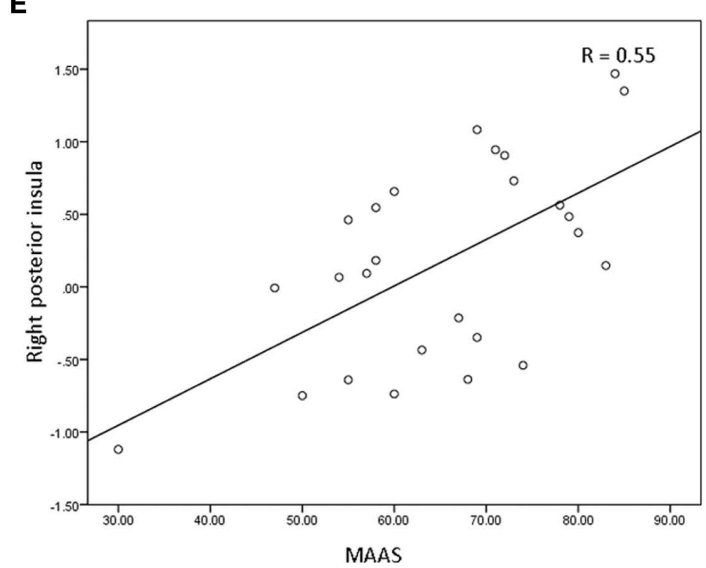

FIGURE 3 | Meditation-specific activity in posterior insula. (A) Significant interaction effect in meditators compared with controls in response to unfair offers (\$19:1-\$15:5) relative to fair offers (\$10:10-\$14:6). Activity was significant in right posterior insula for this contrast. (B) Average beta estimates from 29 voxels in the right posterior insula during the 6-s period following the offer are plotted according to subsequent decision to accept or reject unfair offers (\$19:1-\$15:5). Activity in this region is driven by unfair offers that are rejected relative to accepted in meditators. In contrast, no such response is significant in

tators. So, when playing the Ultimatum Game, meditators were distinct from controls not only in their decision-making behavior, but also in its underlying neural correlates. However, although the meditators played the game more like the rational homo economicus, they did not draw upon the network of lateral prefrontal and parietal regions typically seen for mathematical and logical reasoning (Duncan et al., 2000; Goel and Dolan, 2003; Lee et al., 2006).

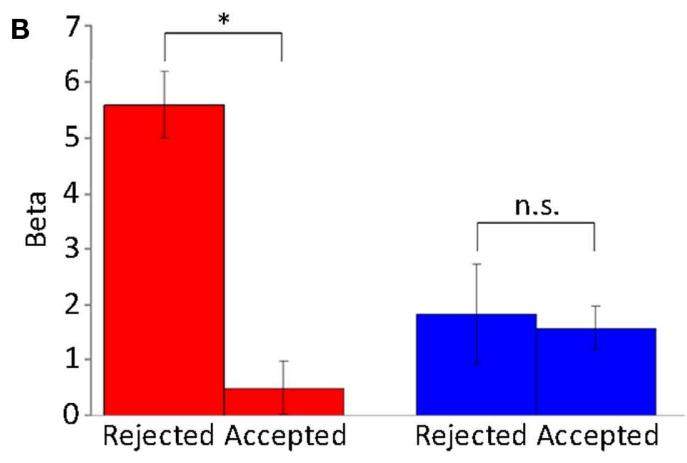

D

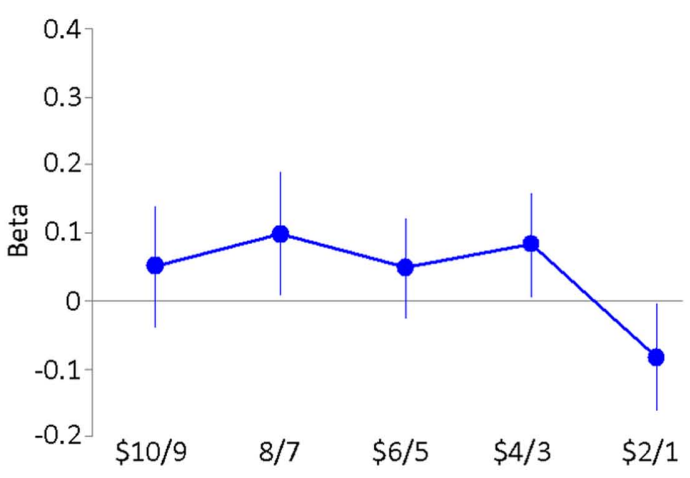

$\mathbf{F}$

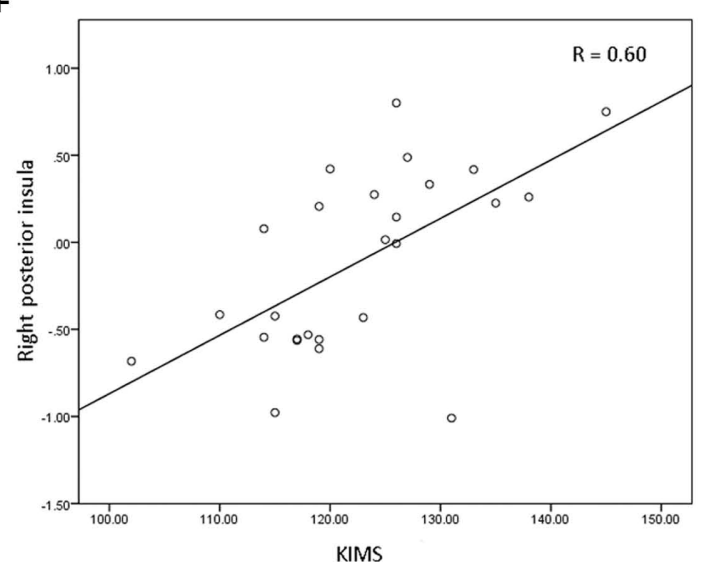

controls. (C,D) Average beta estimates from the voxels in the right posterior insula. The plots display a linear scaling with size of unfairness in meditators, which is not present in controls. Mean \pm SE are plotted in increments of $\$ 2$ bins. Activity within the right posterior insula in meditators is significantly correlated with individual differences in trait mindfulness levels as measured by (E) the Mindfulness Attention Awareness Scale (MAAS) and (F) the Kentucky Inventory of Mindfulness Skills (KIMS). The Pearson correlation coefficient (R) is given in the plot. Each data point represents a subject.

Instead, they drew upon the posterior insula and thalamus: areas usually linked to visceral, emotional rather than rational, deliberative functions.

The behavioral difference between meditators and controls was significant for the most asymmetric offers (Figure 1C). Focusing on unfair trials, a direct contrast of activation between the two groups showed illuminating differences in neural activity (Figures 2C,D). 


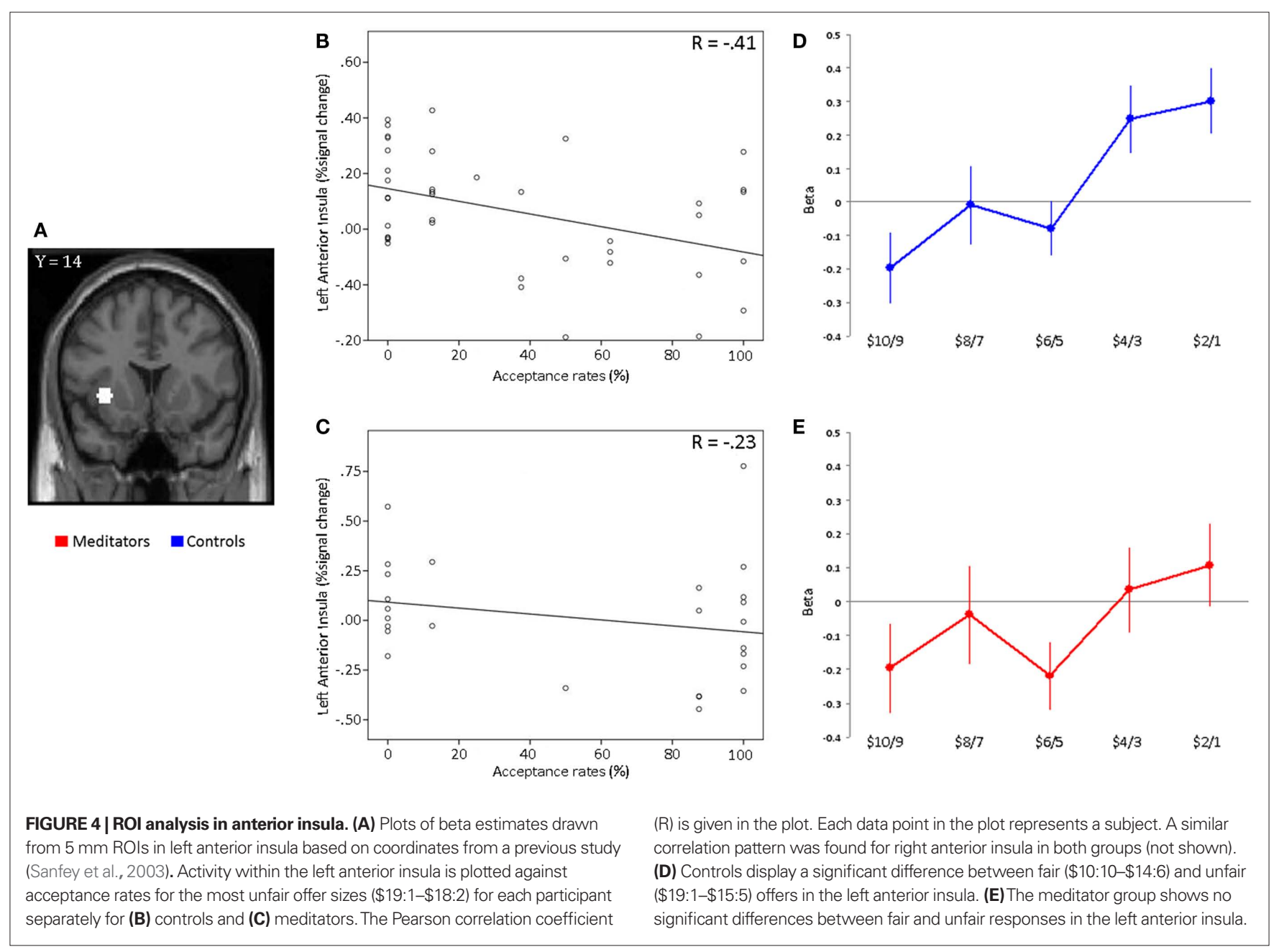

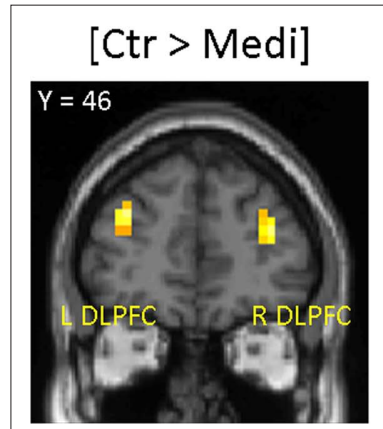

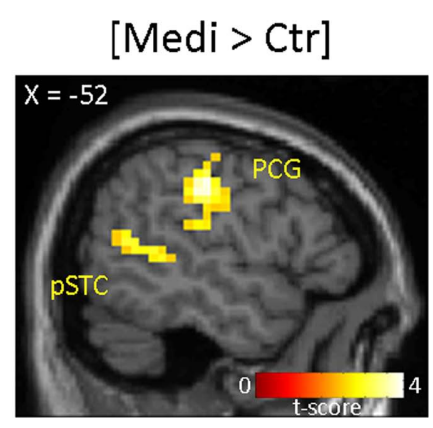

FIGURE 5 | Comparison between a subset of participants that play the Ultimatum Game like rational agents; controls $(n=9)$ and meditators $(\boldsymbol{n}=\mathbf{1 4})$. The main effect [Controls $>$ Meditators] display elevated activity in bilateral DLPFC, whereas in the opposite contrast [Meditators > Controls] the left postcentral gyrus (PCG) and left posterior superior temporal cortex (pSTC) are displayed.

Controls responded to asymmetric offers by engaging the precuneus and a dorsal region of the caudate nucleus. This specific region of the precuneus figures prominently in studies of episodic memory and prospection (the construction of imaginary or future personal scenarios; Buckner and Carroll, 2007; Addis et al., 2009; Spreng et al., 2009). The dorsal caudate region also appears as a specific neural correlate of "fictive error," or the difference between actual and optimal reward, during financial decision-making (Lohrenz et al., 2007). Hence, control subjects may be assessing asymmetric offers in terms of past or hypothetical future scenarios and fictive losses. Furthermore, the dorsal striatum is elevated during altruistic punishment of defectors in an economic exchange (de Quervain et al., 2004), presumably reflecting signals to punish norm violators, which in the current study may explain controls' increasing rejection rates to the most asymmetric offers, compared with meditators. In contrast, meditators showed greater activity in the insula and PPC: areas more closely linked to interoception and attending to the present moment (Critchley et al., 2004; Farb et al., 2007). There exist physiological evidence from previous meditation studies that the left insula is predominantly responsible for parasympathetic control (Lutz et al., 2009; Tang et al., 2009), whereas the right insula, which we observed in the interaction between group (meditator versus control) and offer type (unfair versus fair; Figure 3), has in several studies been proposed to play a role in attending to internal bodily states (Craig, 2003). Previous meditation studies have found right insula involvement in focused attention to internal experi- 
Table 2 |Within group main effects of unfairness for human conditions [meditators unfair $>$ meditators fair].

\begin{tabular}{lrrrrr}
\hline \multirow{2}{*}{ Region } & Voxels $\boldsymbol{t}$-Value & \multicolumn{2}{c}{ MNI } \\
\cline { 4 - 6 } & & & $\boldsymbol{x}$ & $\boldsymbol{y}$ & $\boldsymbol{z}$ \\
\hline Left ACC (BA 32) & & & -4 & 44 & 8 \\
Left mid/anterior insula & 24 & 3.80 & -36 & 4 & 8 \\
Right posterior insula & 12 & 3.55 & -36 & -24 & 16 \\
Left posterior insula & 83 & 4.58 & 32 & -24 & 16 \\
Right postcentralgyrus & 89 & 4.13 & -32 & -24 \\
Thalamus: & 11 & 4.02 & 20 & -44 & 68 \\
Right ventroposterior lateral nucleus & & 3.80 & 16 & -20 & 4 \\
Right ventral lateral nucleus & & 3.43 & 16 & -16 & 12 \\
Right/left medial dorsal nucleus & & 3.65 & 4 & -12 & 4 \\
Left cerebellum & 20 & 4.82 & -8 & -52 & -8
\end{tabular}

Activations are displayed at $p<0.05$, FDR-corrected. Extent threshold $>10$ voxels. AAC, anterior cingulate cortex; BA, Brodmann area.

Table 3 | Within group main effects of unfairness for human conditions [controls unfair $>$ controls fair].

\begin{tabular}{|c|c|c|c|c|c|}
\hline \multirow[t]{2}{*}{ Region } & \multirow[t]{2}{*}{ Voxels } & \multirow[t]{2}{*}{$t$-Value } & \multicolumn{3}{|c|}{ MNI } \\
\hline & & & $x$ & $y$ & $z$ \\
\hline Right IFG/anterior insula & 49 & 4.29 & 32 & 20 & -8 \\
\hline Left IFG/anterior insula & 50 & 3.83 & -44 & 24 & -8 \\
\hline Right medial frontal gyrus (BA 9/10) & 22 & 5.44 & 8 & 60 & 16 \\
\hline Left medial frontal gyrus (BA 10) & 11 & 5.16 & -8 & 60 & 20 \\
\hline Right ACC (BA 24/32) & 21 & 4.40 & 8 & 28 & 24 \\
\hline Left ACC (BA 32) & 15 & 4.22 & -4 & 36 & 24 \\
\hline Right superior frontal gyrus (BA 8) & 18 & 4.82 & 16 & 32 & 56 \\
\hline Right superior temporal sulcus (STS) & 69 & 4.52 & 48 & -28 & -8 \\
\hline Left superior temporal sulcus (STS) & 44 & 4.37 & -52 & -40 & -4 \\
\hline Right supramarginal gyrus & 75 & 5.70 & 60 & -52 & 28 \\
\hline Right cerebellum & 14 & 4.52 & 24 & -76 & -32 \\
\hline
\end{tabular}

Activations are displayed at $p<0.05$, FDR-corrected. Extent threshold $>10$ voxels. IFG, inferior frontal gyrus; $A A C$, anterior cingulate cortex; $B A$, Brodmann area. ences (Holzel et al., 2008), and momentary self-reference (Farb et al., 2007). Based on these findings it is likely that meditators particularly during unfair offers were better able than controls to maintain interoceptive awareness, e.g., attending to internal bodily states. This interpretation is further supported by the finding that posterior insula is significantly correlated with behavioral measures of mindfulness trait levels in meditators in the direction of being more engaged in those meditators with higher mindfulness scores as measured by the MAAS and KIMS.

In the subset of participants who tended to accept the most unfair offers we found increased activity in bilateral DLPFC for controls, presumably reflecting the higher cognitive demands in order to overcome the emotional tendency to reject an unfair offer (Figure 5). The involvement of the DLPFC in playing the Ultimatum Game like homo economicus is well known (Sanfey et al., 2003; Knoch et al., 2006). This result is also consistent with recent studies associating the DLPFC with self-control (Hare et al., 2009) which provide an anatomical base for successful self-regulation in rational controls. Yet the subset of rational meditators displayed activity in a different set of regions suggesting that they were not motivated by economic self-interest. This group recruited the somatosensory cortex, pSTC, and the parahippocampal gyrus. The somatosensory cortex is reported in studies requiring mapping of subjective feeling states arising from bodily responses (Critchley et al., 2004; Lutz et al., 2008b, 2009). Whereas the pSTC is involved in shifting attention to focus on another's perspective (Behrens et al., 2008; Hampton et al., 2008) as well as related to altruistic behavior (Harbaugh et al., 2007; Tankersley et al., 2007).

\section{Table 5 | Demographic variables of controls and meditators.}

\begin{tabular}{lcc}
\hline & Controls $(\boldsymbol{n}=\mathbf{4 0})$ & Meditators $(\boldsymbol{n = 2 6 )}$ \\
\hline Age & $36.8(10.1)$ & $40.4(10.4)$ \\
Female:male & $21: 19$ & $10: 16$ \\
SES & $49.9(8.4)$ & $48.3(12.1)$ \\
Meditation experience (years) & - & $9.5(7.8)$ \\
\hline
\end{tabular}

Mean demographic variables were compared using two-sample t-tests assuming unequal variance. SD in parentheses. No significant differences $(p<0.05)$ between controls and meditators were found. SES; social economic status.

Table 4 | Group $\times$ offer type (unfair, fair) interactions for human conditions.

\begin{tabular}{|c|c|c|c|c|c|}
\hline \multirow[t]{2}{*}{ Region } & \multirow[t]{2}{*}{ Voxels } & \multirow[t]{2}{*}{$t$-Value } & \multicolumn{3}{|c|}{ MNI } \\
\hline & & & $x$ & $y$ & $z$ \\
\hline Right posterior insula & 29 & 3.82 & 48 & -28 & 20 \\
\hline Right postcentral gyrus (BA 3) & 16 & 3.79 & 20 & -44 & 72 \\
\hline
\end{tabular}

Activations are displayed at $p<0.001$, uncorrected. Extent threshold $>10$ voxels. ${ }^{*}$ Significant at $p<0.05$ after whole brain cluster correction with a t-threshold of 3.1 and an extent of 36 voxels. 
Meditators and controls also showed a marked difference in the activity of the anterior insula during the Ultimatum Game. The anterior insula has previously been linked to the emotion of disgust (Calder et al., 2001), and plays a key role in social norm violations, rejection, betrayal, and mistrust (Rilling et al., 2002; Spitzer et al., 2004; KingCasas et al., 2005; Montague and Lohrenz, 2007). In previous studies of the Ultimatum Game, anterior insula activity was higher for unfair offers, and the strength of its activity predicted the likelihood of an offer being rejected (Sanfey et al., 2003). In the present study, this was true for controls, but not for meditators. In control subjects, the anterior insula became active in response to unfair offers, and individuals with higher anterior insula activity tended to reject more of such offers (Figures 4B,D). However, in meditators, the anterior insula showed no significant activation for either fair or unfair offers, and there was no significant relationship between anterior insula activity and offer rejection (Figures 4C,E). Hence, meditators were able to uncouple the negative emotional response to an unfair offer, presumably by attending to internal bodily states reflected by activity in the posterior insula. This relationship was not apparent in control subjects. Meditators may not experience unfair offers as social norm violations, as suggested by their higher acceptance rates for asymmetric offers.

One limitation of the present study is that it employed a crosssectional rather than longitudinal design. Hence, it was not possible to compare the behavior of the subjects before and after they started practicing meditation. Without this information, we cannot yet determine whether the meditators actually acquired a different behavioral profile through meditation experience, or whether the meditation group is simply a highly selected subset of a rare behavioral phenotype within the general population. Future work may help to determine whether a structured program of meditative training can produce the observed changes in social cognition and decision-making.

To summarize, we have identified a population of human beings with an unusual tendency to behave more like rational economic agents in the Ultimatum Game. Specifically, these experienced meditators are roughly twice as likely as controls to say yes to a

\section{REFERENCES}

Addis, D. R., Pan, L., Vu, M. A., Laiser, N., and Schacter, D. L. (2009). Constructive episodic simulation of the future and the past: distinct subsystems of a core brain network mediate imagining and remembering. Neuropsychologia 47, 2222-2238.

Adolphs, R. (2009). The social brain: neural basis of social knowledge. Annu. Rev. Psychol. 60, 693-716.

Amodio, D. M., and Frith, C. D. (2006). Meeting of minds: the medial frontal cortex and social cognition. Nat. Rev. Neurosci. 7, 268-277.

Ariely, D. (2008). Predictably Irrational. New York, NY: HarperCollins.

Baer, R. A., Smith, G. T., and Allen, K. B. (2004). Assessment of mindfulness by report. The Kentucky inventory of mindfulness skills. Assessment 11, 191-206.

Behrens, T. E., Hunt, L. T., Woolrich, M. W., and Rushworth, M. F. (2008).

free dollar over no money at all, rather than reject the reward due to its unfavorable social context. Proximately, meditators seem to avoid generating aversive responses in the anterior insula during unfair offers. In controls, such responses are powerful predictors of rejecting offers during social interaction, but in meditators, these responses are largely absent during the Ultimatum Game. Future studies should assess whether blunting of the high-level emotional representation of the anterior insula is an automatic interoceptive response based on acquired mindfulness skills. Our results suggest that the lower-level interoceptive representation of the posterior insula is recruited based on individual trait levels in mindfulness. When assessing unfair offers, meditators seem to activate an almost entirely different network of brain areas than do normal controls. Controls draw upon areas involved in theory of mind, prospection, episodic memory, and fictive error. In contrast, meditators instead draw upon areas involved in interoception and attention to the present moment.

The rejection of asymmetric rewards is often seen as an important tool for enforcing social norms and encouraging cooperative behavior (Fehr and Gachter, 2002). Unfortunately, it can also have the opposite effect. Siblings, schoolchildren, and CEOs have all been known to worry more about their competitors' rewards than their own - with unhappy social consequences for everyone else. This study suggests that the trick may lie not in rational calculation, but in steering away from what-if scenarios, and concentrating on the interoceptive qualities that accompany any reward, no matter how small.

\section{ACKNOWLEDGMENTS}

We would like to thank Nathan Apple and Mark Ross for help scripting the experiments. Krystle Bartley, Carrie Howard, and Christine Cortelyou for data collection. Claudia Bracero for administrative assistance. This work was supported by grants from the Kane Family Foundation (P. Read Montague), the National Institute of Health (RO1-DA11723; RO1-MH085496, P. Read Montague).

Craig, A. D. (2003). In teroception: the sense of the physiological condition of the body. Curr. Opin. Neurobiol. 13, 500-505.

Craig, A. D. (2009). How do you feel now? The anterior insula and human awareness. Nat. Rev. Neurosci. 10, 59-70.

Creswell, J. D., Way, B. M., Eisenberger, N. I., and Lieberman, M. D. (2007). Neural correlates of dispositional mindfulness during affect labeling. Psychosom. Med. 69, 560-565.

Critchley, H. D., Wiens, S., Rotshtein, P.A., and Dolan, R. J. (2004). Neural systems supporting interoceptive awareness. Nat. Neurosci. 7, 189-195.

Crockett, M. J., Clark, L., Tabibnia, G., Lieberman, M. D., and Robbis, T. W. (2008). Serotonin modulates behavioural reactions to unfairness. Science 320, 1739.

de Quervain, D. J., Fischbacher, U., Treyer, V., Schellhammer, M., Schnyder, U.,
Buck, A., and Fehr, E. (2004). The neural basis of altruistic punishment. Science 305, 1254-1258.

Deichmann, R., Gottfried, J. A., Hutton, C., and Turner, R. (2003). Optimized EPI for $\mathrm{AMRI}$ studies of the orbitofrontal cortex. Neuroimage 19, 430-441.

Duncan, J., Seitz, R. J., Kolodny, J., Bor, D., Herzog, H., Ahmed, A., Newell, F. N., and Emslie, H. A. (2000). Neural basis for general intelligence. Science 289, 457-460.

Eippert, F., Veit, R., Weiskopf, N., Erb, M., Birbaumer, N., and Anders, S. (2007). Regulation of emotional responses elicited by threat-related stimuli. Hum. Brain Mapp. 28, 409-423.

Farb, N. A., Segal, Z. V., Mayberg, H., Bean, J., McKeon, D., Fatima, Z., and Anderson, A. K. (2007). Attending to the present: mindfulness meditation reveals distinct neural modes of selfreference. Soc. Cogn. Affect. Neurosci. 2, 313-322. 
Fehr, E., and Fischbacher, U. (2003). The nature of human altruism. Nature 425 , 785-791.

Fehr, E., and Gachter, S. (2002). Altruistic punishment in humans. Nature 415, 137-140.

Friston, K. J., Fletcher, P., Joseph, O., Holmes, A., Rugg, M. D., and Turner, R. (1998). Event-related fMRI: Characterizing differential responses. Neuroimage 7, 30-40.

Friston, K. J., Williams, R., Howard, R. S., Frackowiak, R. S. J., and Turner, R. (1996). Movement-related effects in fMRI time-series. Magn. Reson. Med. 35, 346-355.

Glaser, D., and Friston, K. J. (2004). "Variance components," in Human Brain Function, eds R. S. J. Frackowiak, K. J. Friston, C. D. Frith, R. J. Dolan, C. J. Price, and S.Zeki (Elsevier Academic Press), 781-793.

Goel, V., and Dolan, R. J. (2003). Reciprocal neural response within lateral and ventral medial prefrontal cortex during hot and cold reasoning. Neuroimage 20, 2314-2321.

Greene, J. D., Nystrom, L. E., Engell, A. D., Darley, J. M., and Cohen, J. D. (2004). The neural bases of cognitive conflict and control in moral judgment. Neuron 44, 389-400.

Guth, W., Schmittenberger, R., and Schwarze, B. (1982). An experimental analysis of ultimatum bargaining. J. Econ. Behav. Organ. 3, 376.

Hampton, A. N., Bossaerts, P., and O'Doherty, J. P. (2008). Neural correlates of mentalizing-related computations during strategic interactions in humans. Proc. Natl. Acad. Sci. U.S.A. 105, 6741-6746.

Harbaugh, W. T., Mayr, U., and Burghart, D. R. (2007). Neural responses to taxation and voluntary giving reveal motives for charitable donations. Science 316, 1622-1625.

Hare, T., Camerer, C. F., and Rangel, A. (2009). Self-control in decisionmaking involves modulation of the VMPFC valuation system. Science 324, 646-648.

Holzel, B. K., Ott, U., Gard, T., Hempel, H., Weygandt, M., Morgen, K., and Vaitl, D. (2008). Investigation of mindfulness meditation practitioners with voxel-based morphometry. Soc. Cogn. Affect. Neurosci. 3, 55-61.
Jackson, D. C., Malmstadt, J. R., Larson, C. L., and Davidson, R. J. (2000). Suppression and enhancement of emotional responses to unpleasant pictures. Psychophysiology37,515-522.

Jensen, K., Call, J., and Tomasello, M. (2007). Chimpanzees are rational maximizers in an ultimatum game. Science 318, 107-109.

King-Casas, B., Sharp, C., Lomax-Bream,L., Lohrenz, T., Fonagy, P., and Montague, P. R. (2008). The rupture and repair of cooperation in borderline personality disorder. Science 321, 806-810.

King-Casas, B., Tomlin, D., Anen, C., Camerer, C. F., Quartz, S. R., and Montague, P. R. (2005). Getting to know you: reputation and trust in a two-person economic exchange. Science 308, 79-83.

Knoch, D., Pascual-Leoner, A., Meyer, K., Treyer, V., and Fehr, E. (2006). Diminishing reciprocal fairness by disrupting the right prefrontal cortex. Science 314, 829-832.

Koenigs, M., and Tranel, D. (2007). Irrational economic decision-making after ventromedial prefrontal damage: evidence from the ultimatum game. J. Neurosci. 27, 951-956.

Lazar, S. W., Kerr, C. E., Wasserman, R. H., Gray, J. R., Greve, D. N., Treadway, M. T., McGarvey, M., Quinn, B. T., Dusek, J. A., Benson, H., Rauch, S.L., Moore, C.I., and Fischl, B. (2005). Meditation experience is associated with increased cortical thickness. Neuroreport 16, 1893-1897.

Lee, K. H., Choi, Y. Y., Gray, J. R., Cho, S. H., Chae, J. H., Lee, S., and Kim, K. (2006). Neural correlates of superior intelligence: stronger recruitment of posterior parietal cortex. Neuroimage 29, 578-586.

Lohrenz, T., McCabe, K., Camerer, C. F., and Montague, P. R. (2007). Neural signature of fictive learning signals in a sequential investment task. Proc. Natl. Acad. Sci. U.S.A. 104, 9493-9498.

Luders, E., Toga, A. W., Lepore, N., and Gaser, C. (2009). The underlying anatomical correlates of long-term meditation: larger hippocampal and frontal volumes of gray matter. Neuroimage 45, 672-678.

Lutz, A., Greischar, L. L., Perlman, D., and Davidson, R. J. (2009). BOLD signal in insula is differentially related to cardiac function during compassion meditation in experts vs novices. Neuroimage 47, 1038-1046.

Lutz, A., Slagter, H. A., Dunne, J. D., and Davidson, R. J. (2008a). Attention regulation and monitoring in meditation. Trends Cogn. Sci. (Regul. Ed.) 12, 163-169.

Lutz, A., Brefczynski-Lewis, J. A., Johnstone, T., and Davidson, R. J. (2008b). Voluntary regulation of the neural circuitry of emotion by compassion meditation: effects of expertise. PLoS ONE3, e1897. doi: 10.1371/ journal.pone.0001897

McClure, S.M.,Laibson, D. I., Loewenstein, G., and Cohen, J. D. (2004). Separate neural systems value immediate and delayed monetary rewards. Science 306, 503-507.

Montague, P. R., and Lohrenz, T. (2007). To detect and correct: norm violations and their enforcement. Neuron 56, 14-18.

Novak, M. A., Page, K. M., and Sigmund, K. (2000). Fariness versus reason in the ultimatum game. Science 289, 1773-1775.

Ochsner, K. N., Bunge, S. A., Gross, J. J., and Gabrieli, J. D. (2002). Rethinking feelings: an FMRI study of the cognitive regulation of emotion. J. Cogn. Neurosci. 14, 1215-1229.

Ochsner, K. N., and Gross, J. J. (2005) The cognitive control of emotion. Trends Cogn. Sci. (Regul. Ed.) 9, 242-249.

Ogawa, S., Lee, T. M., Kay, A. R., and Tank, D. W. (1990). Brain magnetic resonance imaging with contrast dependent on blood oxygenation. Proc. Natl. Acad. Sci. U.S.A. 87, 9868-9872.

Rilling, J., Gutman, D., Zeh, T., Pagnoni, G., Berns, G., and Kilts, C. (2002). A neural basis for social cooperation. Neuron 35, 395-405.

Sanfey, A. G., Rillig, J. K., Aronson, J. A., Nystrom, L. E., and Cohen, J. D. (2003). The neural basis of economic decision-making in the ultimatum game. Science 300, 1755-1758.

Spitzer, M., Fischbacher, U., Herrnberger, B., Grön, G., and Fehr, E. (2004). The neural signature of social norm compliance. Neuron 56, 185-196.

Spreng, R. N., Mar, R. A., and Kim, A. S. (2009). The common neural basis of autobiographical memory, prospection, navigation, theory of mind, and the default mode: a quantitative meta-analysis. J. Cogn. Neurosci. 21, 489-510.

St. Evans, J. (2008). Dual-processing accounts of reasoning, judgment, and social cognition. Annu. Rev. Psychol. 59, 255-278.

Tang, Y.Y., Ma, Y., Fan, Y., Feng, H., Wang, J., Feng, S., Lu, Q., Hu, B., Lin, Y., Li, J., Zhang, Y., Wang, Y., Zhou, L., and Fan, M. (2009). Central and autonomic nervous system interaction is altered by short-term meditation. Proc. Natl. Acad. Sci. U.S.A. 106, 8865-8870.

Tankersley, D., Stowe, C. J., and Huettel, S. A. (2007). Altruism is associated with an increased neural response to agency. Nat. Neurosci. 10, 150-151.

Thaler, R. H. (1988). Anomalies: the ultimatum game. J. Econ. Perspect. 2, 195-206.

Tversky, A., and Kahneman, D. (1981). The framing of decisions and the psychology of choice. Science 211, 453-458.

van't Wout, M., Kahn, R. S., Sanfey, A. G., and Aleman, A. (2006). Affective state and decision-making in the ultimatum game. Exp. Brain Res. 169, 564-568.

Conflict of Interest Statement: The authors declare that the research was conducted in the absence of any commercial or financial relationships that could be construed as a potential conflict of interest.

Received: 07 December 2010; accepted: 24 March 2011; published online: 18 April2011. Citation: Kirk U, Downar J and Montague PR (2011) Interoception drives increased rational decision-making in meditators playing the ultimatum game. Front. Neurosci. 5:49. doi: 10.3389/ fnins.2011.00049

This article was submitted to Frontiers in Decision Neuroscience, a specialty of Frontiers in Neuroscience.

Copyright (C) 2011 Kirk, Downar and Montague. This is an open-access article subject to a non-exclusive license between the authors and Frontiers Media SA, which permits use, distribution and reproduction in other forums, provided the original authors and source are credited and other Frontiers conditions are complied with. 